\title{
Evaluating the fitness of combinations of adsorbents for quantitative gas sensor arrays
}

\author{
Rachel Sousa ${ }^{1}$ and Cory M. Simon ${ }^{* 2}$ \\ ${ }^{1}$ Department of Mathematics. Oregon State University. Corvallis, OR. \\ ${ }^{2}$ School of Chemical, Biological, and Environmental Engineering. Oregon State \\ University. Corvallis, OR. \\ *Cory.Simon@oregonstate.edu
}

September 26, 2020

\begin{abstract}
Robust, high-performance gas sensing technology has applications in industrial process monitoring and control, air quality monitoring, food quality assessment, medical diagnosis, and security threat detection. Nanoporous materials (NPMs) could be utilized as recognition elements in a gas sensor because they selectively adsorb gas. Imitating mammalian olfaction, sensor arrays of NPMs use measurements of the adsorbed mass of gas in a set of distinct NPMs to infer the gas composition. Modular and adjustable NPMs, such as metal-organic frameworks (MOFs), offer a vast materials space to sample for combinations to comprise a sensor array that produces a response pattern rich with information about the gas composition.

Herein, we frame quantitative gas sensing, using arrays of NPMs, as an inverse problem, which equips us with a method to evaluate the fitness of a proposed combination of NPMs in a sensor array. While the (routine) forward problem is to use an adsorption model to predict the mass of gas adsorbed in the NPMs when immersed in a gas mixture of a given composition, the inverse problem is to predict the gas composition from the observed mass of adsorbed gas in each NPM. The fitness of a given combination of NPMs for gas sensing is then determined by the conditioning of its inverse problem: the prediction of the gas composition provided by a fit (unfit) combination of NPMs is insensitive (sensitive) to inevitable errors in the measurements of the mass of gas adsorbed in the NPMs. For illustration, we use experimentally measured adsorption data to analyze the conditioning of the inverse problem associated with a [IRMOF-1, HKUST-1] $\mathrm{CH}_{4} / \mathrm{CO}_{2}$ sensor array.
\end{abstract}

\section{Introduction}

New, robust, high-performance gas sensing technology [1]2] would find applications in worker safety and process control in industry [3], air quality monitoring [4, 5], food quality assessment [6] 7], the diagnosis of disease [8], and security threat (chemical warfare agents, explosives) detection [9] 10]. 
Gas sensors can produce qualitative or quantitative predictions about the composition of a gas. Qualitative gas sensors aim to discriminate between distinctive and, often, complex gas mixtures, e.g. between the breath of healthy patients and of lung cancer patients [11]. Quantitative gas sensors, the subject of this article, aim to determine the concentrations of components in a gas mixture, e.g. of carbon monoxide in the air of a home [12].

Nanoporous materials, such as metal-organic frameworks (MOFs) [13], covalent organic frameworks (COFs) [14], porous organic cage molecules (POCs) [15], and metal-organic polyhedra (MOPs) [16], could be utilized as recognition elements in a gas sensor by exploiting their selective gas adsorption properties [17-23]. In principle, any measurable property of a nanoporous material that is altered by the adsorption of gas- such as mass [24], luminescence [25], mechanical strain [26, 27], conductivity [28], or color [29]- could be exploited to infer changes in the gas composition. Without much loss of generalization, we focus on utilizing a MOF as a sensor by monitoring the mass of adsorbed gas in it [18]. The mass of adsorbed gas depends upon and thus contains information about the composition of the bulk gas in which the MOF is immersed; given a mathematical model of gas adsorption, observation of the adsorbed mass of gas in a MOF places a constraint on the composition of the gas phase. A mass-based MOF sensor can be miniaturized by depositing a thin film [30-32] of the MOF on a quartz crystal microbalance (QCM) [33] or surface acoustic wave (SAW) device [34], which can measure the (total) mass of gas adsorbed in the thin film at the nano-gram scale [18]. Several experimental feasibility studies demonstrated gas sensing with MOF-coated QCMs [24, 35-42] and SAWs [43-45] for binary gas mixtures or distinct, pure gases.

Engineering MOFs that diversely interact with the different components of the gas is paramount for the development of MOF-based sensors. Attributed to their modular synthesis (building blocks: metal nodes/clusters and organic linker molecules) [46] and post-synthetic modifiability [47], many MOFs with diverse pore sizes, shapes, and surface chemistries, and therefore with diverse interactions with gases, can be and have been synthesized [48].

Any individual mass-based MOF sensor, however, is insufficient for quantitative gas sensing in domains where the gas mixture has multiple degrees of freedom (e.g., gas mixtures whose components vary independently in concentration) because of cross-sensitivity. Practically, many components of a gas mixture will adsorb in the MOF and contribute to the adsorbed mass (although, to varying extents). Consequently, any given (total) adsorbed mass in the MOF could result from many different compositions, rendering the single-sensor response $\rightarrow$ gas composition problem non-invertible.

MOF-based gas sensor arrays [49] confront cross-sensitivity by using measurements of the adsorbed mass of gas in multiple, distinct MOFs to infer the gas composition. See Fig. 1. Despite that each MOF in the array could adsorb all components of the gas mixture (cross-sensitivity), the collection of adsorbed mass measurements in each MOF in the array (the response pattern) could provide sufficient information to determine the composition of a multi-component mixture, i.e., to render the sensor array response pattern $\rightarrow$ gas composition problem invertible. In other words, a cross-sensitive gas sensor array is capable of producing an information-rich, high-dimensional response pattern from which we can determine the concentrations of several components in multi-component gas mixtures encountered in practice [49]. The modularity and adjustability of MOFs allows us to synthesize a diverse set of MOFs to comprise a sensor array that produces a response pattern with high infor- 
mation content. Inspiringly, a sensor array comprised of cross-sensitive MOFs is analogous to the mammalian olfactory system (see Ref. [50], Box 1), whose olfactory receptors are cross-sensitive to different odorants [51]; in part for this reason, a sensor array in conjunction with a pattern recognition system [52] is dubbed an "electronic nose" [1 [53].

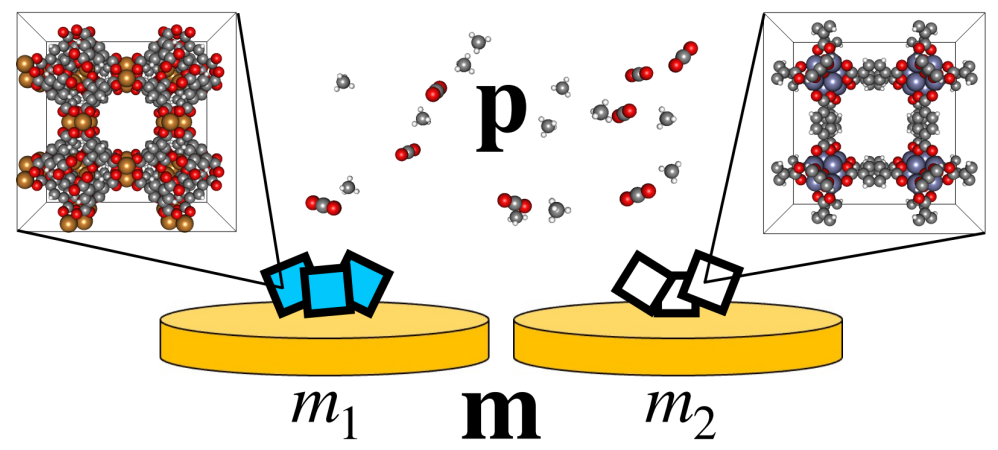

Figure 1: A gas sensor array that exploits the selective gas adsorption properties of metal-organic frameworks (MOFs). Two distinct MOFs [HKUST-1, IRMOF-1] are in equilibrium with a $\mathrm{CH}_{4} / \mathrm{CO}_{2}$ gas mixture, with $\mathbf{p}=\left[p_{\mathrm{CO}_{2}}, p_{\mathrm{CH}_{4}}\right]$ the partial pressures of its components. The total mass of gas adsorbed in each MOF, $\mathbf{m}=\left[m_{1}, m_{2}\right]$, depends on $\mathbf{p}$. The forward problem, routine in the porous materials community, is to predict $\mathbf{m}$ from $\mathbf{p}$. In quantitative gas sensing, the task is to solve the inverse problem: predict $\mathbf{p}$ from $\mathbf{m}$. However, the observation of $\mathbf{m}$ is inevitably contaminated by measurement error that propagates onto and corrupts the predicted gas composition. The fitness of a proposed combination of MOFs for a gas sensor array depends on the sensitivity of the predicted gas composition to this measurement error.

The computational design of MOF-based gas sensor arrays is an emerging research area pioneered by Wilmer and coworkers [50,54-57]. Instead of choosing a combination of MOFs for a sensor array by trial-and-error, computational methods that can rank combinations of MOFs for a gas sensor array, based on either simulated [58] or experimentally measured adsorption properties, can accelerate the development and deployment of MOF-based gas sensor arrays.

In this article, to enable the computational design of quantitative gas sensor arrays, we formulate the problem of determining the composition of a multi-component gas mixture using mass-based MOF sensor arrays as an applied [59] inverse problem [60-62]. The (isothermal) forward problem associated with a set of MOFs immersed in a gas mixture is routine in the porous materials community: given the gas composition and an adsorption model, determine the mass of gas adsorbed in each MOF at thermodynamic equilibrium. The adsorption model could be constructed from experimental adsorption measurements [63 64], molecular simulations of adsorption [58], simple statistical mechanical models [65], or statistical machine learning models (e.g., a neural network [66]). The inverse problem, defined in relation to the forward problem, is encountered in gas sensing: given the mass of gas adsorbed in each MOF and an adsorption model, determine the gas composition. While the forward problem is concerned with determining the effect (gas adsorption) of the cause (gas composition), the inverse problem is concerned with determining the cause from the effect']

${ }^{1}$ To justify invoking causality here, we take the gas phase to be a reservoir so that the mass of gas adsorbed in the MOF cannot change the composition of the gas phase. 
The formulation of mass-based MOF array sensing as an inverse problem provides a methodology to rank combinations of MOFs for a gas sensor array based on their adsorption properties (more precisely, based on a mixed-gas adsorption model for each MOF in the array). When solving the inverse problem, by inverting the adsorption model, inevitable noise/error in the mass measurements propagates onto and corrupts the prediction of the gas composition; the optimal gas sensor array will yield an inverse problem whose solution, the predicted gas composition, is insensitive/robust to this measurement error [50,59].

\section{Inverse problems}

The most famous inverse problem [59.67] in mathematics is perhaps "can one hear the shape of a drum?" [68-70]. Here, we provide an illustrative example of an inverse problem that is more accessible to the chemical sciences.

Consider a hot cup of coffee solution at a (spatially uniform) initial temperature, $T_{0}$. The temperature of the coffee, $T=T(t)$, evolves with time, $t$, as it exchanges heat with the air at ambient temperature, $T_{a}$. Take $T_{0}, T_{a} \in\left(0^{\circ} \mathrm{C}, 100^{\circ} \mathrm{C}\right)$ so that the coffee begins and remains in the liquid phase. A crude dynamic model for $T=T(t)$ is:

$$
\begin{aligned}
& C \frac{d T}{d t}=h A\left(T_{a}-T\right) \\
& T(t=0)=T_{0}
\end{aligned}
$$

with $C$ the thermal capacity of the coffee, $h$ the heat transfer coefficient, and $A$ the surface area of the coffee through which heat is transferred. Take the time constant $\tau:=C /(h A)$ and $T_{a}$ as known. In the forward problem, the initial temperature $T_{0}$ is given, and the task is to use this dynamic model to predict the temperature at a later time $t^{*}>0, T\left(t^{*}\right)$. Fig. 2 shows the numerical solution to eqn. 1 for varying $T_{0}$ in eqn. 2 In an associated inverse problem, the temperature at a later time $t^{*}>0$, $T\left(t^{*}\right)$, is given, and the task is use the model to predict the initial temperature, $T_{0}$ [71]. Graphically, the solution to the inverse problem is found in Fig. 2 by identifying the point $\left(t^{*}, T\left(t^{*}\right)\right)$ on the graph and following the trajectory that passes through it backwards in time to reach $T_{0}$. Though simple, this inverse problem presents interesting features:

(inversion of causality) In the forward problem, we predict the effect (later in time) of a cause (earlier in time), whereas, in the inverse problem, we predict the cause of an effect.

$\square$ (ill-conditioning) For $t^{*}>>\tau$, the temperature of the coffee, $T(t)$, is close to the ambient air temperature, $T_{a}$, for all initial temperatures, $T_{0}$. As a result, for $t^{*}>>\tau$, a small error in the measurement of $T\left(t^{*}\right)$ is amplified into a large change in the predicted $T_{0}$. For $t^{*}$ sufficiently large, it is practically impossible to predict $T_{0}$ from $T\left(t^{*}\right)$ accurately because of this ill-conditioning.

(possibility that a solution does not exist) For $t^{*}>>\tau$, small errors in the measured $T\left(t^{*}\right)$ could result in a predicted $T_{0}$ outside the feasible range of temperatures for which the model is valid, $T \in\left(0^{\circ} \mathrm{C}, 100^{\circ} \mathrm{C}\right)$.

N.b., model error emanating from simplifying assumptions to arrive at eqn. 11also corrupts the prediction of $T_{0}$ in the inverse problem. 


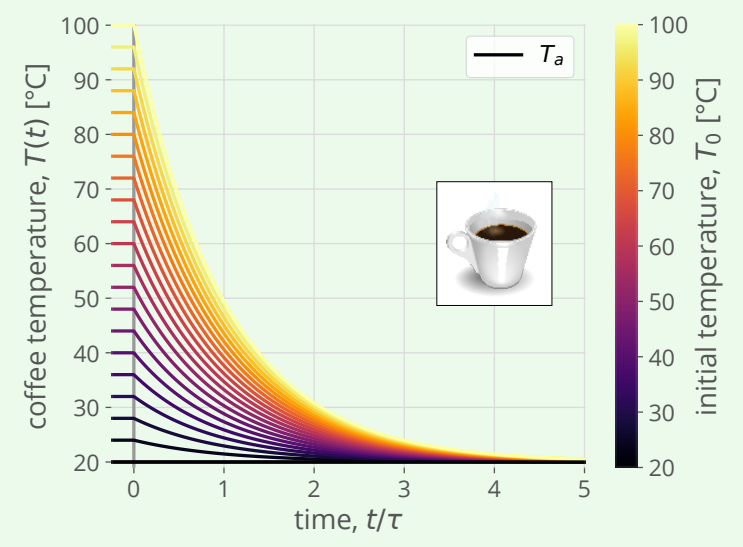

Figure 2: The temperature $T(t)$ of coffee at different initial temperatures $T_{0}$ under the model in eqns. 1 1 2 with time constant $\tau:=C /(h A)$ and $T_{a}=20^{\circ} \mathrm{C}$.

\section{Framing an electronic nose as an applied inverse problem}

We first frame quantitative gas sensing, using measurements of the mass of gas adsorbed in an array of MOFs, as a mathematical inverse problem [60-62].

Consider a set of $n_{m}$ distinct MOFs in thermodynamic equilibrium with a gas mixture containing $n_{g}$ components. Let $\mathbf{p} \in \mathbb{R}^{n_{g}}$ denote the gas composition- a vector containing the partial pressure of each component of the gas mixture- and $T$ be the temperature of the gas. Let $\mathbf{m} \in \mathbb{R}^{n_{m}}$ denote the resulting sensor array response- a vector containing the total adsorbed mass of gas in each MOF.

\subsection{The adsorption model}

The adsorption model

$$
f(\mathbf{p} ; T)=\mathbf{m}
$$

maps the composition $\mathbf{p}$ and temperature $T$ of the gas phase to the equilibrium mass of gas adsorbed in each MOF, m. Hereafter, we take temperature as fixed and omit $T$ in eqn. 3 making $f$ the equilibrium, mixed-gas adsorption isotherm. We assume the domain of $f$ is comprised of all possible gas compositions, $\mathcal{P} \equiv[0, \infty)^{n_{g}}$. The codomain of $f$ is $\mathcal{M} \equiv[0, \infty)^{n_{m}}$. i.e. $f: \mathcal{P} \rightarrow \mathcal{M}$.

In contrast to our previous study [50], we allow $f$ to be nonlinear to account for (i) the saturation of adsorption sites in the MOF and (ii) non-additive contributions to the adsorbed mass of gas by different components of the gas. We take $f: \mathbf{p} \mapsto \mathbf{m}$ to be a continuously differentiable function, thereby prohibiting adsorption/desorption hysteresis and gas-induced structural transitions seen in flexible MOFs [72].

Constructing an adsorption model is routine in the porous materials community, whether through fitting/interpolating experimental adsorption data [63], ideal adsorbed solution theory [73-75], molec- 
ular models and simulations of gas adsorption [58], simple statistical mechanical models [65], or statistical machine learning models [66].

\subsection{The forward problem}

The forward problem is also routine- and sometimes trivial- in the porous materials community: given the gas composition $\mathbf{p}$ and the adsorption model $f$, determine the equilibrium mass of gas adsorbed in each MOF, $\mathbf{m}$. Per the constraints we imposed on $f$, which prohibit adsorption/desorption hysteresis, the solution to the forward problem exists and is unique.

\subsection{The (applied) inverse problem}

In quantitative gas sensing, we are confronted with the inverse problem, defined in relation to the forward problem: given the mass of gas adsorbed in each MOF, $\mathbf{m}$, and the adsorption model, $f$, determine the gas composition $\mathbf{p}$. i.e., the inverse problem is to find $\mathbf{p}$ that satisfies $\mathbf{m}=f(\mathbf{p})$.

As opposed to an exact inverse problem, we consider the practically relevant applied inverse problem [59], where we take into account uncertainties in the inputs. In practice, the observations of the adsorbed mass of gas in each MOF are corrupted by measurement noise/error, $\delta \mathbf{m} \in \mathbb{R}^{n_{m}}$. This measurement error will propagate onto and corrupt the prediction of the gas composition. Acknowledging this is key to evaluating the fitness of a gas sensor array: the optimal gas sensor array will yield an inverse problem whose solution, the prediction of the gas composition, is insensitive to the measurement error.

However, despite the well-posedness of the forward problem, the applied inverse problem could have (i) multiple solutions if $f$ is not injective (one-to-one) or (ii) no solution if $f$ is not surjective (onto). An underdetermined system, giving case (i), certainly occurs if the array is composed of an insufficient number of MOFs to fully constrain the $n_{g}$ degrees of freedom in the gas composition $\left(n_{m}<n_{g}\right)$. Case (ii) is problematic if measurement error contaminates $\mathbf{m}$ and pushes it outside the range of $f$. The inverse problem has no solution if $\mathbf{m}+\delta \mathbf{m} \notin \mathcal{M}^{\prime} \equiv f(\mathcal{P})$. This almost certainly occurs if there are more MOFs comprising the sensor array than degrees of freedom in the gas composition $\left(n_{m}>n_{g}\right)$, giving an overdetermined system. See Ref. [50] for further discussion in the context of linear systems.

Hereafter, we assume the sensor array is composed of $n_{m}=n_{g}$ distinct MOFs (i) to avoid under- and over-determined systems, which will require a longer paper to treat, and (ii) as the most economical yet sufficient gas sensor array. Moreover, we take the adsorption model $f$ to be injective so that, if a solution to the inverse problem exists, it is unique.

\subsection{A remark on causality}

Changes in the gas composition cause changes in the amount of gas adsorbed in the MOFs, provided the gas phase is a reservoir. For this reason, adsorption models $f(\mathbf{p} ; T)$ are often constructed from underlying physical principles (thermodynamics) [58 65. 73], but are sometimes empirical [66]. While 
the task in the forward problem is to determine the effect $(\mathbf{m})$ of the cause (p), in the inverse problem, the task is to determine the cause (p), given knowledge of its effect (m).

\subsection{A left inverse of the adsorption model}

The applied inverse problem has a unique solution provided (i) $f$ is injective and (ii) the observed mass vector falls in the range of $f$, i.e., $\mathbf{m}+\delta \mathbf{m} \in \mathcal{M}^{\prime}=f(\mathcal{P})$. We find the solution by constructing the left inverse of the adsorption model, $g: \mathcal{M}^{\prime} \rightarrow \mathcal{P}$ :

$$
g(\mathbf{m}):=f^{-1}(\mathbf{m})=\mathbf{p} \Longleftrightarrow f(\mathbf{p})=\mathbf{m} .
$$

We carefully defined the domain of the left inverse $g$ to be the image of $f, \mathcal{M}^{\prime}$. From the adsorption model $f$, we can (i) possibly, analytically solve for $g(\mathbf{m})$ or (ii) numerically, for a given $\mathbf{m}$, compute $g(\mathbf{m})=\mathbf{p}$ by searching for a root [76] of the function $f(\mathbf{p})-\mathbf{m}$.

\subsection{The conditioning of the applied inverse problem}

The conditioning of an inverse problem refers to the sensitivity of its solution to a perturbation in the input [61]. In an ill-conditioned (well-conditioned) problem, small perturbations in the input cause large (small) perturbations in the solution. In quantitative gas sensing, the input is the observed/measured sensor array response, and the solution is the predicted gas composition. We wish the inverse problem in gas sensing to be well-conditioned, so that errors in the measured mass of adsorbed gas in each MOF minimally corrupt the predicted gas composition.

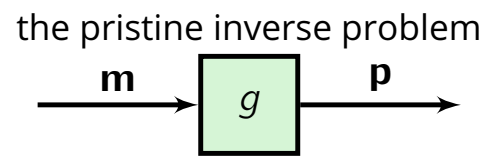

the perturbed inverse problem

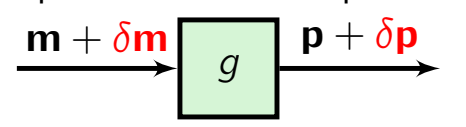

Figure 3: To analyze the conditioning of the inverse problem, we consider when the pristine input, $\mathbf{m}$, is contaminated by measurement error, $\delta \mathbf{m}$. The solution to this perturbed problem differs from the solution to the pristine problem by $\delta \mathbf{p}$, by its definition. We wish $\|\delta \mathbf{p}\|$ to be small, so that the predicted gas composition is insensitive to measurement error. The resulting $\|\delta \mathbf{p}\|$ depends on $g$, which in turn depends on the combination of MOFs that comprise the sensor array.
Let $\delta \mathbf{m} \in \mathbb{R}^{n_{m}}$ be a perturbation in the sensor array response, $\mathbf{m}$. Assume that (i) $f$ is injective, so that its left inverse, $g$ in eqn. 4. exists and (ii) the observed response falls in the image of $f$, i.e., $\mathbf{m}+\delta \mathbf{m} \in \mathcal{M}^{\prime}$. In the pristine inverse problem, the input is $\mathbf{m}$, and the predicted gas composition is $\mathbf{p}=g(\mathbf{m})$. In the perturbed inverse problem, the input is $\mathbf{m}+\delta \mathbf{m}$, and the predicted gas composition is $\mathbf{p}+\delta \mathbf{p}=g(\mathbf{m}+\delta \mathbf{m})$, which defines $\delta \mathbf{p}$ as the perturbation of the pristine solution that results from the perturbation $\delta \mathbf{m}$ of the pristine input. See Fig. 3. The conditioning of the inverse problem is characterized by the ratio of the relative error in the predicted gas composition to the error in the mass measurements (since we generally expect $\|\delta \mathbf{p}\|$ to grow with $\|\delta \mathbf{m}\|)$ :

$$
\frac{\|\delta \mathbf{p}\| /\|\mathbf{p}\|}{\|\delta \mathbf{m}\|}=\frac{\|g(\mathbf{m}+\delta \mathbf{m})-g(\mathbf{m})\| /\|\mathbf{p}\|}{\|(\mathbf{m}+\delta \mathbf{m})-\mathbf{m}\|}
$$


The equality on the right in eqn. 5 shows conditioning as the ratio of the relative error in the output of $g$ to the error in the input to $g$ (see Fig. 3).

The condition number [77] is defined by eqn. 5 under the limit of small $\|\delta \mathbf{m}\|$, allowing us to make a linear approximation:

$$
g(\mathbf{m}+\delta \mathbf{m}) \approx g(\mathbf{m})+\mathbf{J}_{g}(\mathbf{m}) \delta \mathbf{m},
$$

with $\mathbf{J}_{g}=\mathbf{J}_{g}(\mathbf{m})$ the Jacobian matrix of the left inverse function, $g$. The condition number is then:

$$
\chi:=\lim _{\epsilon \rightarrow 0^{+}} \sup _{\|\delta \mathbf{m}\| \leq \epsilon} \frac{\|\delta \mathbf{p}\| /\|\mathbf{p}\|}{\|\delta \mathbf{m}\|}=\frac{\left\|\mathbf{J}_{g}\right\|_{o p}}{\|\mathbf{p}\|},
$$

and it quantifies the sensitivity of the predicted gas composition to small perturbations in the measurements of mass adsorbed in each MOF comprising the sensor array. The supremum in eqn. 7 is needed because, generally, $\|\delta \mathbf{p}\|$ depends on not only the magnitude of $\delta \mathbf{m}$, but on its direction as well. The equality on the right of eqn. 6. with $\|\cdot\|_{o p}$ the operator norm, follows from the definition of the operator norm of $\mathbf{J}_{g}$.

The condition number $\chi$ in eqn. 7 has a geometric interpretation. Under the linear approximation in eqn. 6. the perturbation in the gas composition is a linear transformation of the (small) perturbation of the measured masses, $\delta \mathbf{p} \approx \mathbf{J}_{g}(\mathbf{m}) \delta \mathbf{m}$. The operator norm of the Jacobian matrix, $\left\|\mathbf{J}_{g}\right\|_{o p}$, is the maximum factor by which the vector $\delta \mathbf{m}$ can be stretched by transforming it with $\mathbf{J}_{g}$. For a wellconditioned inverse problem, we wish $\left\|\mathbf{J}_{g}\right\|_{o p}$ to be small to minimize the stretching of $\delta \mathbf{m}$ when it is transformed into $\delta \mathbf{p}$.

N.b. the condition number $\chi$ generally depends on $\mathbf{m}$, i.e., $\chi=\chi(\mathbf{m})$, because $\mathbf{J}_{g}=\mathbf{J}_{g}(\mathbf{m})$. We may also say that $\chi$ depends on the gas composition to which the sensor array is exposed, since $\chi(\mathbf{m})=\chi(f(\mathbf{p}))$.

Finally, we link the conditioning of the inverse problem directly to the properties of the adsorption model, $f$, using the inverse function theorem. The Jacobian matrix of $g$ evaluated at $\mathbf{m}$ is equal to the matrix inverse of the Jacobian of $f$ evaluated at $\mathbf{p}=g(\mathbf{m})$, giving $\chi$ viewed as a function of $\mathbf{p}$ :

$$
\chi=\chi(\mathbf{p})=\frac{\left\|\left[\mathbf{J}_{f}(\mathbf{p})\right]^{-1}\right\|_{o p}}{\|\mathbf{p}\|}
$$

Because (i) the operator norm of a matrix is equal to its largest singular value and (ii) the singular values of the inverse of a matrix, if it exists, are obtained by inverting the singular values of the matrix [78], $\chi$ is equal to the inverse of the smallest singular value of $\mathbf{J}_{f}(\mathbf{p})$ divided by $\|\mathbf{p}\|$. Of course, the ordering of the MOFs/gases in the vectors $\mathbf{m} / \mathbf{p}$ is immaterial, since the singular values of $\mathbf{J}_{f}(\mathbf{p})$ are invariant to permutations of the rows and columns.

\subsection{Evaluating the fitness of a gas sensor array}

The condition number, $\chi$ in eqn. 7. quantifies the fitness of a proposed combination of MOFs to compose a gas sensor array. It describes the sensitivity of the predicted gas composition to errors in the measurements of the mass of gas adsorbed in the MOFs. We can determine $\chi$ from the adsorption 
model, $f(\mathbf{p})$, that governs gas adsorption in the proposed combination of MOFs via eqns. 4 and 8 . Because $\chi=\chi(\mathbf{m})=\chi(f(\mathbf{p}))$, the fitness of a sensor array depends upon the gas composition to which it is exposed.

\subsection{Remark on absolute versus relative errors}

We can define four condition numbers based on combinations of the relative and absolute error in the input and output of $g$ [60]. We opted to define the condition number in eqn. 7 using the relative error in the gas composition, $\|\delta \mathbf{p}\| /\|\mathbf{p}\|$ and the absolute error in the measured masses, $\|\delta \mathbf{m}\|$. To justify $\|\delta \mathbf{p}\| /\|\mathbf{p}\|$, e.g., $\|\delta \mathbf{p}\|=0.01$ bar may be acceptable if the partial pressures are on the order of 100 bar, but unacceptable if on the order of 0.1 bar. The suitability of using absolute error in $\mathbf{m}$ depends upon the noise/error characteristics of the (QCM or SAW) device that measures the mass of adsorbed gas in the MOFs. If the typical error $\|\delta \mathbf{m}\|$ scales with $\|\mathbf{m}\|$, relative error is more appropriate. Our work can easily be adapted to work with relative errors in $\|\mathbf{m}\|$. N.b., when using a condition number to compare the fitness of two sensor arrays operating in the same gas composition, defining the conditioning in terms of absolute or relative error in $\mathbf{p}$ produces equivalent rankings.

\section{Examples of the analysis of conditioning of the inverse prob- lem}

We framed the problem of predicting gas composition from observation of the adsorbed masses of gas in an array of MOFs as an applied inverse problem. Now, we illustrate how this framework allows us to quantify and rank the fitness of combinations of MOFs for gas sensor arrays. For case studies, we consider single-MOF methane sensing and double-MOF methane/carbon dioxide sensing. The two candidate materials are canonical MOFs, HKUST-1 [79] and IRMOF-1 [80], in which we have experimentally measured, pure- $\mathrm{CH}_{4}$ and pure- $\mathrm{CO}_{2}$ adsorption data at $298 \mathrm{~K}$ [64 81]. See Fig. 4 . Note that we use g/g units for $\mathbf{m}$, assuming an equivalent mass of each MOF is used in the array.

In the determined inverse problems we analyze below, the number of (distinct) MOFs in the array $n_{m}$ is equal to the number of components of the gas $n_{g}$, and each observation of a mass of gas adsorbed in a MOF of the array restricts one degree of freedom in the gas composition.

The code and data to reproduce all plots in this article are available at github . com/SimonEnsemble/ nonlinear_sensing.

\subsection{A single-MOF sensor array}

A single-MOF $\left(n_{m}=1\right)$ sensor for a pure gas $\left(n_{g}=1\right)$ is an instructive starting point. Consider either HKUST-1 or IRMOF-1 in thermodynamic equilibrium with a bath of pure methane gas at temperature $T$ and pressure $p$. 


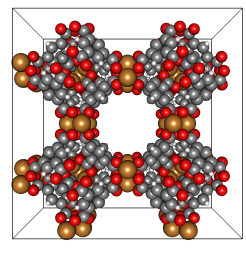

(a) HKUST-1

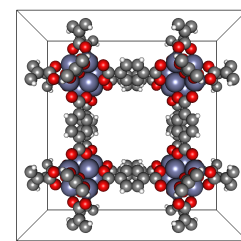

(b) IRMOF-1

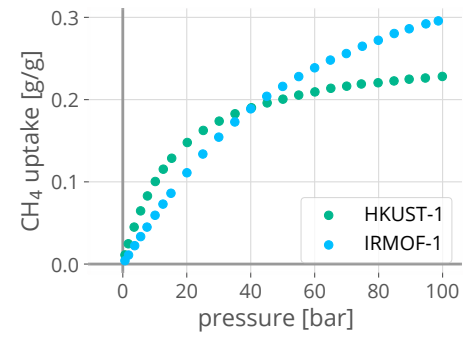

(c) $\mathrm{CH}_{4}$ adsorption data

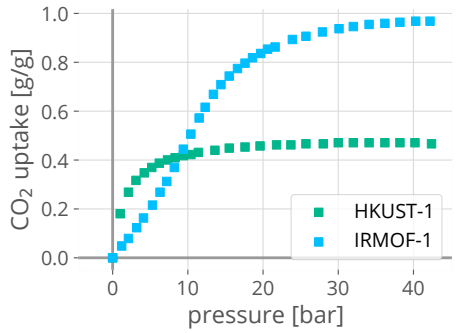

(d) $\mathrm{CO}_{2}$ adsorption data

Figure 4: Experimental methane and carbon dioxide adsorption data in IRMOF-1 and HKUST-1 to illustrate analysis of the conditioning of the inverse problem associated with the gas sensor array depicted in Fig. 1. (a, b) Crystal structures of HKUST-1 [79] and IRMOF-1 [80]. (c, d) Experimentally measured, pure-component, equilibrium methane [64] and carbon dioxide [81] uptake at $298 \mathrm{~K}$ in HKUST-1 and IRMOF-1. Note difference in scales on the axes.

The adsorption model. We use the Langmuir adsorption model [65] to describe the adsorption of methane in each MOF, at fixed temperature, as a function of pressure:

$$
m=f(p ; T)=m_{\infty} \frac{K p}{1+K p},
$$

where $m_{\infty}=\lim _{p \rightarrow \infty} f(p)$ is the saturation loading and $K=K(T)$ is the Langmuir parameter that describes the affinity of gas for the MOF ( $p=K^{-1} \Longrightarrow m=m_{\infty} / 2$ ). We identify the parameters $K$ and $m_{\infty}$ for both HKUST-1 and IRMOF-1 by least-squares fitting to the experimental methane adsorption isotherm data at $T=298 \mathrm{~K}$. Fig. 5 a displays the (good) Langmuir model fits to the data. The domain and image of $f$ in eqn. 9 are $\mathcal{P} \equiv[0, \infty)$ and $\mathcal{M}^{\prime} \equiv f(\mathcal{P})=\left[0, m_{\infty}\right)$, respectively. Note that $f(p)$ is injective, but not surjective since $m \leq m_{\infty}$.

The forward problem. The (isothermal) forward problem is to predict the methane uptake in the MOF, $m$, at a given pressure, $p$. The solution to the forward problem is trivial: compute $m=f(p)$ from eqn. 9 with the identified model parameters $K$ and $m_{\infty}$.

The inverse problem. We encounter the (isothermal) inverse problem in methane gas sensing: the task is to, given the mass of methane adsorbed in the MOF, $m$, predict the pressure of the methane gas, $p$, in which the MOF is immersed. While perhaps an academic problem to use a MOF to measure the pressure of a pure gas, this $n_{m}=n_{g}=1$ sensing problem reveals useful insights.

The left inverse of the adsorption model. To find a solution to the inverse problem for input $m \in \mathcal{M}^{\prime}$, we find a closed-form expression for the left inverse of $f$ in eqn. 9

$$
p=g(m):=f^{-1}(m)=\frac{1}{K} \frac{m / m_{\infty}}{1-m / m_{\infty}} .
$$

The domain of the inverse function $g: m \mapsto p$ is $\mathcal{M}^{\prime}=\left[0, m_{\infty}\right)$ and its image is $\mathcal{P}=[0, \infty)$. The left inverse $g(m)$ is shown for HKUST-1 and IRMOF-1 in Fig. $5 b$. 


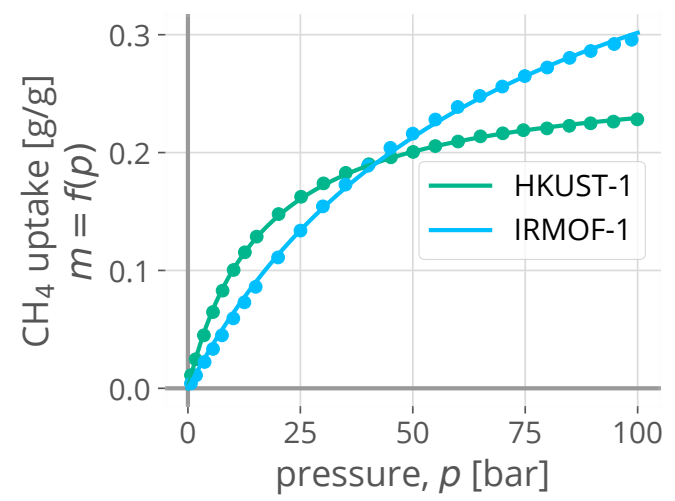

(a)
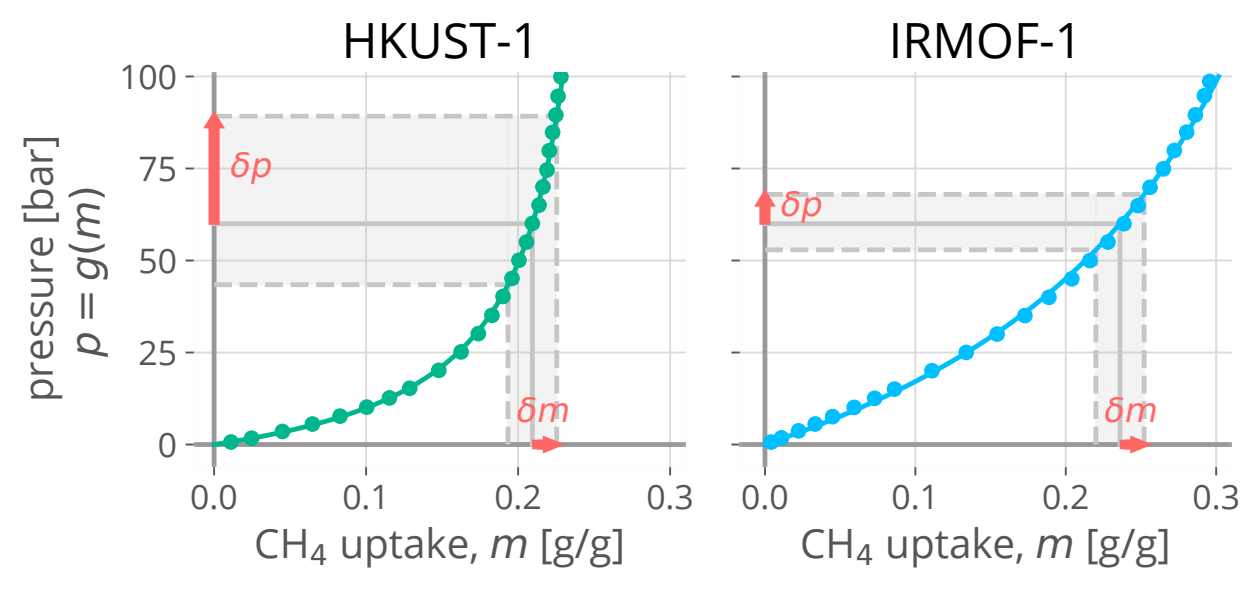

(b)

Figure 5: Methane sensing using a single-MOF sensor, either IRMOF-1 or HKUST-1. (a) The Langmuir adsorption isotherm model $f(p)$ in eqn. 9 (lines) for methane adsorption in HKUST-1 and IRMOF-1 at $298 K$, whose model parameters $K$ and $m_{\infty}$ were identified by fitting to experimental adsorption data (points) from Ref. [64]. (b) Experimental methane adsorption data (points) and left inverse functions $g(m)=f^{-1}(m)$ given in eqn. 10 (green and blue lines) for HKUST-1 and IRMOF-1. Under the scenario that $p=60$ bar (solid, horizontal gray line), the red arrows and gray shaded regions show how a perturbation in the measured adsorbed mass, $\delta m$, the same for both MOFs, perturbs the prediction of the gas pressure by $\delta p$, which is larger in magnitude for HKUST-1.

Notably, measurement error $\delta m$ could push $m+\delta m$ outside the image of $f$ so that the inverse problem has no solution. e.g., if $m+\delta m>m_{\infty}$, then there does not exist a gas composition $p \geq 0$ such that $f(p)=m+\delta m$.

The conditioning of the inverse problem. We now study the conditioning of the inverse problem, which is solved for $m \in \mathcal{M}^{\prime}$ by computing $g(m)$ in eqn. 10 .

Fig. 5b.geometrically illustrates how an error in the measured mass of gas, $\delta m$, propagates onto and 
corrupts the predicted gas composition by $\delta p$. In this scenario, each MOF is immersed in a bath of pure methane gas at $60 \mathrm{bar}$ and $298 \mathrm{~K}$. More methane is adsorbed in IRMOF-1 than in HKUST-1. The measurement error $\delta m$ is equal for both single-MOF sensors. Comparing the resulting perturbation in the predicted gas composition, $\delta p$, between HKUST-1 and IRMOF-1:

The $\delta p$ for HKUST-1 is larger than the $\delta p$ for IRMOF-1; hence, IRMOF-1 has a higher fitness for gas sensing at $p=60$ bar and $298 \mathrm{~K}$ than HKUST-1 because the predicted gas composition is less sensitive to the measurement error.

The magnitude of $\delta p$ depends upon the direction of $\delta m$ in addition to its magnitude.

In the limit of small $\|\delta m\|, \delta p$ is large if $g^{\prime}(m)$ is large, since then a small perturbation in $m$ results in a large perturbation in $p$.

The magnitude of $\delta p$ depends on $p$. At lower pressures, the ranking of the MOFs switches, and HKUST-1 has a higher fitness for methane sensing than IRMOF-1.

The geometric picture in Fig. 5 b reinforces the concept of conditioning and how analysis of conditioning provides a means to juxtapose the fitness of two MOFs for a gas sensor array.

The condition number in eqn. 7 which pertains to small $\|\delta m\|$, becomes the logarithmic derivative of $g$ for a single-MOF sensor,

$$
\chi=\chi(m)=\frac{g^{\prime}(m)}{g(m)}=\frac{1}{m\left(1-m / m_{\infty}\right)},
$$

and for the left inverse function in eqn. 10. The condition number [units: 1/(g/g)] quantifies the perturbation of the predicted pressure of the methane gas that results from small perturbations in the measured adsorbed mass of gas in the MOF. The condition number is more useful when viewed as a function of $p$, since we aim to compare IRMOF-1 and HKUST-1 for sensing at a particular $p$ :

$$
\chi=\chi(p)=\frac{(1+K p)^{2}}{m_{\infty} K p}
$$

Eqn. 12 reveals that the inverse problem is ill-conditioned (large $\chi$ ) at the two extremes of:

low pressures $((K p) \rightarrow 0 \Longrightarrow m \rightarrow 0$ ), because the relative (not absolute) error in the predicted gas composition becomes very large at low pressures. Thus, eqn. 12 recovers the notion of a lower limit of detection.

high pressures $\left((K p) \rightarrow \infty \Longrightarrow m \rightarrow m_{\infty}\right)$, because the pores of the MOF saturate with gas, and the mass of gas adsorbed in the MOF becomes insensitive to changes in gas composition, thereby making the predicted gas composition sensitive to errors in the measured adsorbed mass. Thus, eqn. 12 recovers the notion of an upper limit of detection.

Fig. 6 displays the condition number $\chi$ for both IRMOF-1 and HKUST-1, viewed as a function of $m$ and of $p$ separately. Indeed, the condition number grows unboundedly at high and low pressures for both MOFs. Moreover, the inverse problem is better-conditioned for IRMOF-1 than for HKUST-1 at pressures above ca. 12.5 bar, whereas, below 12.5 bar, HKUST-1 has a lower condition number than IRMOF-1. Fig.6 therefore illustrates that the condition number $\chi$ provides a means to rank the fitness of MOFs for gas sensing at different conditions, given the objective is to choose a MOF that yields a prediction of the gas composition that is insensitive to measurement error. 

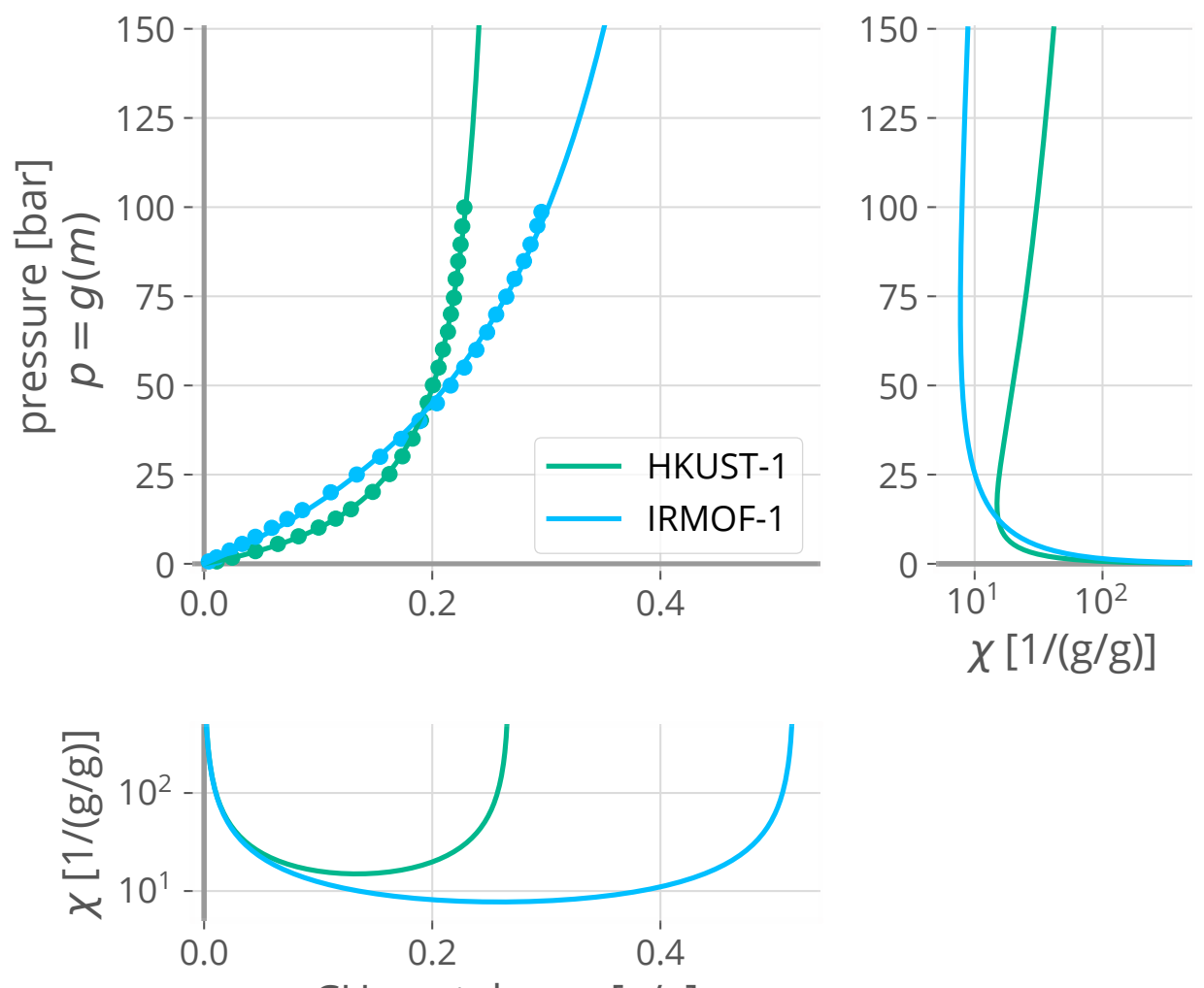

$\mathrm{CH}_{4}$ uptake, $m[\mathrm{~g} / \mathrm{g}]$

Figure 6: The condition number, $\chi$, of the inverse problem for methane sensing using the observed mass of adsorbed gas in either HKUST-1 or IRMOF-1. The upper-left panel shows the inverse function $g(m)$ (line) and experimental data (points) for both HKUST-1 and IRMOF-1. The bottom and right panels show the condition number $\chi$ as a function of $m$ and $p$, respectively, for the two MOFs, given in eqn. 11 and 12. At low (high) pressures, HKUST-1 (IRMOF-1) has a higher fitness for gas sensing because $\chi$ is smaller, hence the predicted pressure of methane is less sensitive to measurement error in $m$.

Finally, we link the condition number, viewed as a function of pressure, directly to the adsorption model $f(p)$ through eqn. 8 .

$$
\chi=\chi(p)=\frac{1}{p f^{\prime}(p)}
$$

This more generally reinforces the conclusions we drew from eqn. 12. (1) as the pressure diminishes, $\chi$ grows unboundedly because the relative error in the pressure blows up while the Henry coefficient, $\lim _{p \rightarrow 0} f^{\prime}(p)$ remains finite and (2) if the slope of the adsorption isotherm, $f^{\prime}(p)$, is small, the condition number is large. The latter inevitably occurs at higher pressures when the pores of the MOF saturate with gas. 


\subsection{A two-MOF sensor array}

We now consider a gas sensor array of $n_{m}=2$ MOFs, IRMOF-1 and HKUST-1, tasked with determining the partial pressures of the $n_{g}=2$ components in a methane/carbon dioxide mixture.

Consider HKUST-1 and IRMOF-1 in thermodynamic equilibrium with a $\mathrm{CH}_{4} / \mathrm{CO}_{2}$ mixture at temperature $T$ with partial pressures $\mathbf{p}=\left[p_{\mathrm{CO}_{2}}, p_{\mathrm{CH}_{4}}\right] \in \mathbb{R}^{2}$. Let $\mathbf{m}=\left[m_{\mathrm{HKUST}-1}, m_{\mathrm{IRMOF}-1}\right] \in \mathbb{R}^{2}$ be the total mass adsorbed in each MOF, comprised of both $\mathrm{CO}_{2}$ and $\mathrm{CH}_{4}$ gas.

The adsorption model. As a mixed-gas $\left(\mathrm{CH}_{4} / \mathrm{CO}_{2}\right)$ adsorption model for each MOF, we invoke Ideal Adsorbed Solution Theory (IAST) [73-75], a thermodynamic framework for predicting mixed-gas adsorption from pure-component (pure $\mathrm{CH}_{4}$ and $\mathrm{CO}_{2}$ ) adsorption isotherms. As input, IAST requires a mathematical model for the pure-component adsorption isotherms in Figs. $4 \mathrm{C}$ and $4 \mathrm{~d}$. For the pure- $\mathrm{CH}_{4}$ adsorption isotherms, we use the fitted Langmuir models (see eqn. 9) shown in Fig. 5 a. We also use a fitted Langmuir model for the pure- $\mathrm{CO}_{2}$ adsorption isotherm in HKUST-1. However, we resort to smoothed cubic spline interpolation of the pure- $\mathrm{CO}_{2}$ adsorption data in IRMOF-1, since the Langmuir model cannot capture the inflection in the data. Fig. \$1 shows all four pure-component adsorption isotherm models on top of the experimental data. The IAST framework, together with the pure-component adsorption models, provide our mixed-gas adsorption model, $\mathbf{m}=f(\mathbf{p} ; T)$, though not explicitly.

The forward problem. The (isothermal) forward problem is to predict the total mass of gas adsorbed, comprised of both $\mathrm{CO}_{2}$ and $\mathrm{CH}_{4}$, in each MOF, $\mathbf{m}$, given the partial pressures of $\mathrm{CO}_{2}$ and $\mathrm{CH}_{4}$ in the gas phase, $\mathbf{p}$.

We find the solution to the forward problem through IAST calculations, implemented in py IAST [74]. i.e., pyIAST provides a numerical implementation of the function $\mathbf{m}=f(\mathbf{p})$.

We visualize the IAST-based adsorption model as a mapping $f: \mathbf{p} \mapsto \mathbf{m}$ in Fig. 70 [50, 82]. The thick, black boundary shows how the square in gas composition space (left) is non-linearly transformed into the more complicated shape in sensor response space (right). Moreover, the vertical/horizontal lines in composition space are transformed into the same-colored vertical-ish/horizontal-ish lines in response space. The images of the vertical lines in composition space have a smaller arc length in response space than the images of the horizontal lines. This indicates that the mass of gas adsorbed in the MOFs is less sensitive to changes in the partial pressure of $\mathrm{CH}_{4}$ than to changes in the partial pressure of $\mathrm{CO}_{2}$; generally $\mathrm{CO}_{2}$ has a higher affinity for MOFs than $\mathrm{CH}_{4}$ owing to its polar bonds. We also can see how the small square regions in composition space, formed by the lines, are mapped into response space. As the partial pressures of the components increase, either together or independently, the images of the (same size) squares in composition space occupy less area in response space. This is because, as the pores of the MOF fill with gas, the mass of gas adsorbed in the MOF becomes less sensitive to charges in the partial pressure.

The inverse problem. We encounter the (isothermal) inverse problem in $\mathrm{CO}_{2} / \mathrm{CH}_{4}$ gas sensing: the task is to, given the total mass of adsorbed gas in each MOF, $\mathbf{m}$, predict the partial pressures of the two components, $\mathbf{p}$, in the gas phase in which the MOFs are immersed. 

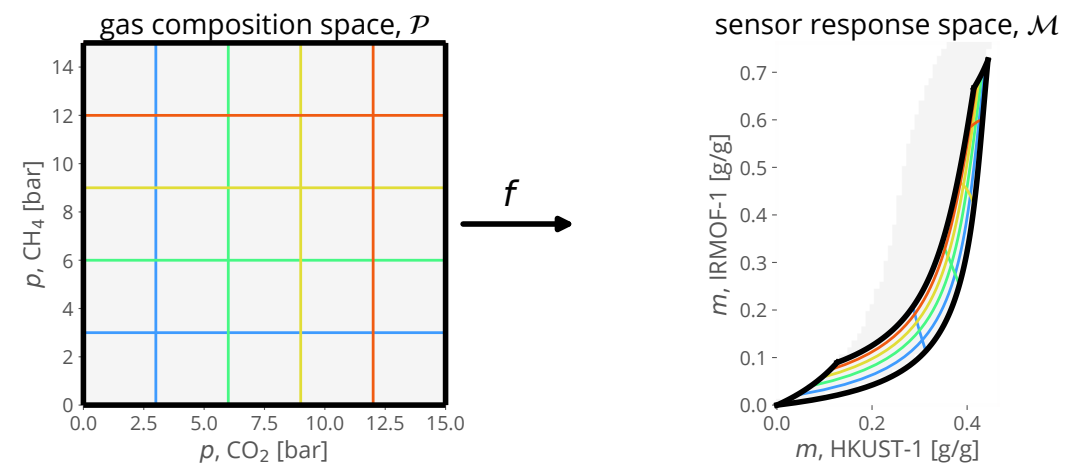

(a)
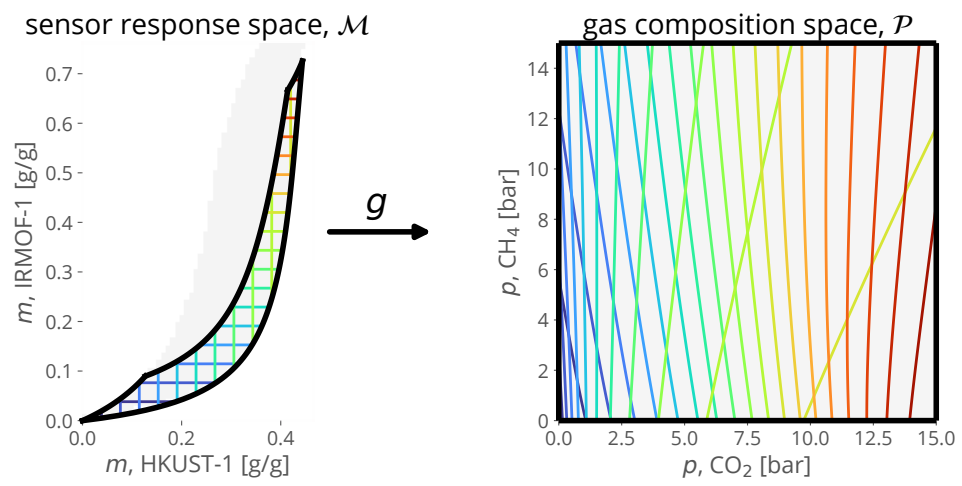

(b)

Figure 7: Visualizing the maps $f: \mathbf{p} \mapsto \mathbf{m}$ and $g: \mathbf{m} \mapsto \mathbf{p}$ for the two-MOF [HKUST-1, IRMOF-1] sensor array immersed in a $\mathrm{CO}_{2} / \mathrm{CH}_{4}$ gas mixture at $298 \mathrm{~K}$. (a) The mapping of composition space into sensor response space by the mixed-gas adsorption model $\mathbf{m}=f(\mathbf{p})$, provided by IAST in conjunction with pure- $\mathrm{CO}_{2}$ and pure- $\mathrm{CH}_{4}$ adsorption isotherm models in Fig. S1. (b) The mapping of sensor response space into gas composition space by the left inverse of the adsorption model, $\mathbf{p}=g(\mathbf{m})$, implemented numerically by a root finding algorithm on the function $f(\mathbf{p})-\mathbf{m}$. For both (a) and (b): The thick, black boundaries gives a global view of how the regions of the domain are transformed by $f$ and $g$ into regions in the codomain. The vertical and horizontal lines of a given color are mapped to the vertical-ish and horizontal-ish lines of the same color. Points outside the gray region in sensor response space are outside the (a) image of $f$ and (b) domain of $g$.

The left inverse of the adsorption model. To implement the left inverse of the adsorption model, $g(\mathbf{m})=\mathbf{p}$, which produces the solution to the inverse problem (if a solution exists), we resort to a numerical root finding algorithm (in SciPy [83]):

$$
\mathbf{p}=g(\mathbf{m})=\text { search for } \mathbf{p} \text { such that } \mathbf{m}-f(\mathbf{p})=\mathbf{0} \text {. }
$$

N.b., sensor array responses that fall outside the gray region in Fig. $7 \mathrm{a}$ are outside the image of $f$; in that case, a solution to the inverse problem does not exist.

We visualize the mapping $g: \mathbf{m} \mapsto \mathbf{p}$ in Fig. $7 \mathrm{~b}$ in a similar manner as in Fig. 7a. The most important 
feature of the mapping is that the (same size) squares in response space are stretched into larger regions in composition space by $g$ as the MOFs adsorb more gas. As a consequence, the inverse problem will be ill-conditioned when the pores of the MOF are nearly saturated with gas; small errors in the measured masses will be stretched into large errors in composition space. Fig 8a illustrates this consequence more clearly.

Fig 8 a shows how circles in response space are mapped into composition space. In response space, the x's represent pristine measurements of $\mathbf{m}$, and the circles around the x's represent perturbed measurements, contaminated by errors $\delta \mathbf{m}$ of the same magnitude. In gas composition space, the same-colored $\mathrm{x}$ represents the true gas composition, with the surrounding oval outline representing the corrupted predictions of the gas composition from using the observations $\mathbf{m}+\delta \mathbf{m}$ to solve the inverse problem. Each colored region in response space represents an uncertainty zone associated with the marked $\mathrm{x}$ : owing to measurement errors, we are unable to confidently distinguish the response $\mathbf{m}$ marked with $\mathrm{x}$ from all responses that fall in its uncertainty zone. As a result, we can at best confidently conclude that the gas composition falls somewhere within the cognate, colored zone in composition space. Fig 8 a reveals, as a result of measurement errors of fixed magnitude $\|\delta \mathbf{m}\|$, the perturbation of the predicted gas composition, $\delta \mathbf{p}$,

depending on the direction of $\delta \mathbf{m}$, could have a much larger component in the direction of $p_{\mathrm{CH}_{4}}$, since the mass of gas adsorbed is less sensitive to changes in $p_{\mathrm{CH}_{4}}$, compared to $p_{\mathrm{CO}_{2}}$. Consequently, the condition number $\chi$ in eqn. 7 has a dominant contribution from the error in the predicted $\mathrm{p}_{\mathrm{CH}_{4}}$.

tends to increase in magnitude as the partial pressures of the gases increase (together or separately). This is a result of the pores of the MOF saturating with gas, causing adsorption to be less sensitive to charges in partial pressure.

The conditioning of the inverse problem. Fig. $8 \mathrm{~b}$ displays the condition number associated with the inverse problem, given in eqn. 8 and viewed as a function of gas composition, i.e., $\chi=\chi(\mathbf{p})$. We computed $\chi(\mathbf{p})$ via (i) numerical differentiation (finite difference method in numdifftools) of $f$ to obtain the Jacobian $\mathbf{J}_{f}(\mathbf{p})$, (ii) computation of the smallest singular value of $\mathbf{J}_{f}(\mathbf{p}), \sigma_{2}$, and then (iii) computation of the operator norm $\left\|\left[\mathbf{J}_{f}(\mathbf{p})\right]^{-1}\right\|_{o p}=\sigma_{2}^{-1}$. As in the $1 \mathrm{D}$ case, the inverse problem is ill-conditioned at low partial pressures (representing the lower limits of detection) and at higher partial pressures of $\mathrm{CO}_{2}$, when the pores of the MOFs saturate with gas. The latter reinforces our observations in Fig. 8 a which shows that measurement errors $\delta \mathbf{m}$ can be stretched into large $\delta \mathbf{p}$ when the partial pressure of $\mathrm{CO}_{2}$ is high.

Gas sensor arrays cope with cross-sensitivity. In gas sensing using QCMs or SAWs, only the total mass of gas is adsorbed, which, for cross-sensitive MOFs, has a contribution from each component of the gas phase. With an adsorption model, however, we have the luxury of showing the adsorption of each component of the gas. Fig. $\mathrm{S} 2$ shows the mass of each component, $\mathrm{CH}_{4}$ and $\mathrm{CO}_{2}$, of the $\mathrm{CH}_{4} / \mathrm{CO}_{2}$ mixture adsorbed in each HKUST-1 and IRMOF-1, as a function of the gas composition, according to IAST. Indeed, HKUST-1 and IRMOF-1 are cross-sensitive MOFs; when exposed to a $\mathrm{CH}_{4} / \mathrm{CO}_{2}$ mixture, each appreciably adsorb both components of the gas. Therefore, observation of the total mass of gas adsorbed in either HKUST-1 or IRMOF-1 in isolation is insufficient to predict the composition of the 

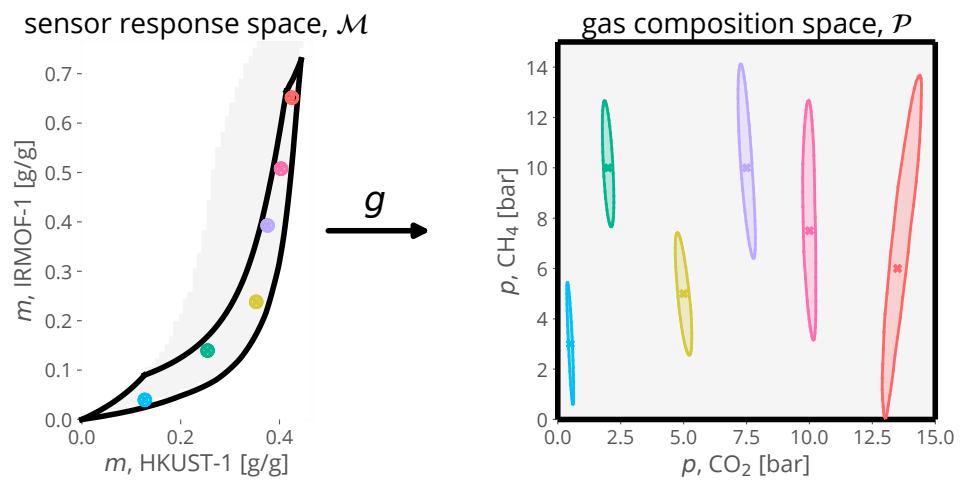

(a)

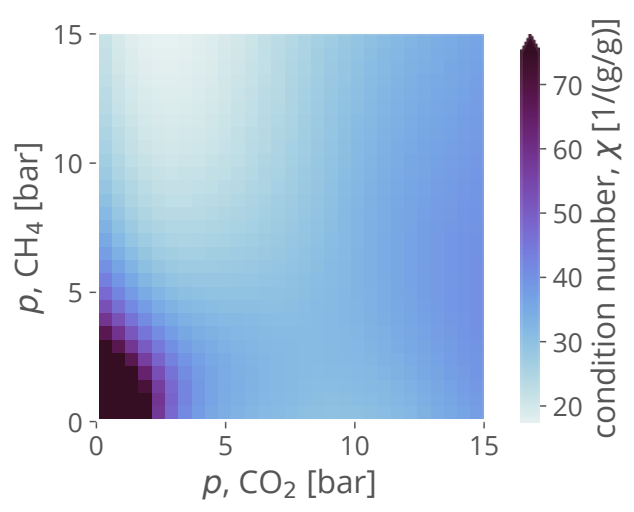

(b)

Figure 8: Conditioning of the inverse problem associated with the double-MOF (HKUST-1, IRMOF-1) $\mathrm{CO}_{2} / \mathrm{CH}_{4}$ sensing array. (a) The circles in sensor response space, all of the same radius, are mapped into the same-colored oval shapes in gas composition space by the left inverse, $g(\mathbf{m})$. This indicates how measurement errors in sensor response space are stretched into composition space. Points outside the gray region in sensor response space are outside the domain of $g$. (b) The condition number $\chi=\chi(\mathbf{p})$ of the inverse problem associated with the two-MOF sensor array [IRMOF-1, HKUST-1] immersed in a $\mathrm{CO}_{2} / \mathrm{CH}_{4}$ mixture.

$\mathrm{CO}_{2} / \mathrm{CH}_{4}$ mixture because e.g., a measurement of $0.05 \mathrm{~g} / \mathrm{g}$ could be due to mostly $\mathrm{CO}_{2}$ or mostly $\mathrm{CH}_{4}$. The total mass of gas adsorbed in both HKUST-1 and IRMOF-1 together, $\mathbf{m}$, however, is sufficient to arrive at a unique prediction of the gas composition, as Fig. $7 \mathrm{~b}$ shows. This underscores the necessity for gas sensor arrays in practical gas sensing applications, where the gas composition has multiple degrees of freedom. Sensor arrays allow us to overcome cross-sensitivity of any individual MOF by allowing each MOF to constrain one degree of freedom in the gas phase; collectively, the collection of responses in the MOFs pinpoint the gas composition. 


\section{Discussion}

We framed quantitative gas sensor arrays, where measurements of the mass of gas adsorbed in an array of adsorbents is used to infer the composition of a gas, as a mathematical inverse problem. The fitness of a proposed combination of adsorbents for quantitative gas sensing is determined by the conditioning of its inverse problem, which is the robustness/insensitivity of the predicted gas composition to errors in the measurements of the adsorbed masses of gas. We demonstrated how the condition number allows us to evaluate and compare the fitness of combinations of adsorbents for gas sensor arrays using a single- and double-MOF sensor array for $\mathrm{CO}_{2} / \mathrm{CH}_{4}$ sensing. The conditioning of the inverse problem depends not only on the adsorption properties of the adsorbents composing the array, but also on the gas composition. In general, the inverse problem becomes ill-conditioned (i, low detection limit) at low pressures because the relative error in the predicted gas composition blows up, and (ii, high detection limit) at high pressures, when the pores of the adsorbent become saturated with gas, causing the mass of gas adsorbed in the MOF to be insensitive to changes in gas composition.

In addition to the mass of adsorbed gas, other measureable properties of MOFs that depend on gas composition can be exploited for gas sensing, e.g., changes in luminescence [25], mechanical strain [26. 27], or conductivity [28]. The ideas in this article, namely quantifying the fitness of a combination of MOFs for a gas sensor array based on the condition number $\chi$, also apply to these other sensing mechanisms, provided that one can construct a model analogous to eqn. 3t that relates the measured property of the MOF (the analogy to $\mathbf{m}$ ) to the gas composition. Exploiting the physical phenomena that "the adsorbed mass of gas in a MOF depends upon the gas composition" to construct a gas sensor is attractive from a computational standpoint because we can, using molecular models and simulations, predict the amount of gas adsorbed in a MOF as a function of gas composition and temperature [58]. This opens the possibility to conduct high-throughout computational screenings of MOF-based electronic noses that exploit the adsorbed mass of gas in the MOFs [54].

Computationally ranking combinations of MOFs for gas sensor arrays is an emerging research area [50.54-57]. At this juncture, we highlight shortcomings of our work and provide new directions.

Our gas sensing framework requires a priori knowledge of what constituents are possibly present in the gas phase. If gas species $X$ is present in the gas phase and adsorbs appreciably in the MOF, but is not accounted for in the adsorption model $f(\mathbf{p})$ in eqn. 3 the prediction of the gas composition using the adsorption model is invalid. Fundamentally, we cannot foresee how to circumvent this, except to, for all gas species that could be present with reasonable probability, incorporate them into the adsorption model.

We studied how measurement error corrupts the prediction of the gas composition, but we ignored model error that emanates from the simplifying assumptions to arrive at the equilibrium gas adsorption model $m=f(p)$. For example, in this work, we took (i) $\mathrm{CH}_{4}$ adsorption to perfectly follow a Langmuir model with the identified $K$ and $m_{\infty}$ and (ii) mixed $\mathrm{CO}_{2} / \mathrm{CH}_{4}$ adsorption to follow Ideal Adsorbed Solution Theory. One way to quantify model error is to study the sensitivity of the predicted gas composition to the model parameters [50].

While we took isothermal conditions in our article, we can adapt our modeling framework to handle 
varying temperatures by constructing a temperature-dependent adsorption model $f(\mathbf{p} ; T)$ as in eqn. 3 then either: (i) input the measured temperature of the gas, identified by an independent measurement, into the adsorption model or (ii) include the temperature as an additional variable to be determined in the inverse problem.

We defined the condition number $\chi$ in eqn. 7 using the absolute error in the measurement, but the expected magnitude of the measurement error could be dependent on the magnitude of the measurement. The noise characteristics of the mass measurement device (QCM or SAW) should be further characterized before sensor design and deployment. For future work, we aim to specify a probabilistic distribution for the errors in the measured masses, $\delta \mathbf{m}$, then determine the distribution of the error in the predicted gas composition, $\delta \mathbf{p}$, that results when solving the inverse problem.

Finally, we aim to study ill-posed inverse problems for future work. In the underdetermined problem, e.g., when there are fewer MOF sensors than gases $\left(n_{m}<n_{g}\right)$, the sensor response is insufficient to fully constrain the gas composition, but (i) it could still provide useful, though incomplete, information about gas composition and (ii) introducing regularization or incorporating a prior belief would alter the inverse problem and give it a unique solution. In the overdetermined problem, when there are more MOF sensors than gases $\left(n_{m}>n_{g}\right)$, we can cast the inverse problem as a least squares problem, as in Ref. [50], giving it a unique solution.

\section{Acknowledgements}

C.M.S acknowledges support from the National Science Foundation (NSF), Award 1920945. Thanks to Arni Sturluson for helpful comments on the manuscript.

\section{References}

[1] Wenwen Hu, Liangtian Wan, Yingying Jian, Cong Ren, Ke Jin, Xinghua Su, Xiaoxia Bai, Hossam Haick, Mingshui Yao, and Weiwei Wu. Electronic noses: from advanced materials to sensors aided with data processing. Advanced Materials Technologies, 4(2):1800488, 2019.

[2] Alphus D Wilson and Manuela Baietto. Applications and advances in electronic-nose technologies. Sensors, 9(7):5099-5148, 2009.

[3] Simone Capone, A Forleo, L Francioso, R Rella, P Siciliano, J Spadavecchia, DS Presicce, and AM Taurino. Solid state gas sensors: state of the art and future activities. Journal of Optoelectronics and Advanced Materials, 5(5):1335-1348, 2003.

[4] Duk-Dong Lee and Dae-Sik Lee. Environmental gas sensors. IEEE Sensors Journal, 1(3):214-224, 2001.

[5] Joshua S Apte, Kyle P Messier, Shahzad Gani, Michael Brauer, Thomas W Kirchstetter, Melissa M Lunden, Julian D Marshall, Christopher J Portier, Roel CH Vermeulen, and Steven P Hamburg. High-resolution air pollution mapping with google street view cars: exploiting big data. Environmental Science \& Technology, 51(12):6999-7008, 2017. 
[6] Emmanuelle Schaller, Jacques O Bosset, and Felix Escher. 'electronic noses' and their application to food. Lebensmittel-Wissenschaft+ Technologie, 31(4):305-316, 1998.

[7] Hanie Yousefi, Hsuan-Ming Su, Sara M Imani, Kais Alkhaldi, Carlos D M. Filipe, and Tohid F Didar. Intelligent food packaging: A review of smart sensing technologies for monitoring food quality. ACS Sensors, 4(4):808-821, 2019.

[8] Gady Konvalina and Hossam Haick. Sensors for breath testing: from nanomaterials to comprehensive disease detection. Accounts of Chemical Research, 47(1):66-76, 2014.

[9] Charles E Davidson, Melissa M Dixon, Barry R Williams, Gary K Kilper, Sung H Lim, Raymond A Martino, Paul Rhodes, Melissa S Hulet, Ronald W Miles, Alan C Samuels, et al. Detection of chemical warfare agents by colorimetric sensor arrays. ACS Sensors, 5(4):1102-1109, 2020.

[10] Jehuda Yinon. Field detection and monitoring of explosives. TrAC Trends in Analytical Chemistry, 21(4):292-301, 2002.

[11] Gang Peng, Ulrike Tisch, Orna Adams, Meggie Hakim, Nisrean Shehada, Yoav Y Broza, Salem Billan, Roxolyana Abdah-Bortnyak, Abraham Kuten, and Hossam Haick. Diagnosing lung cancer in exhaled breath using gold nanoparticles. Nature Nanotechnology, 4(10):669-673, 2009.

[12] G Huyberechts, P Szecowka, J Roggen, and BW Licznerski. Simultaneous quantification of carbon monoxide and methane in humid air using a sensor array and an artificial neural network. Sensors and Actuators B: Chemical, 45(2):123-130, 1997.

[13] Hiroyasu Furukawa, Kyle E Cordova, Michael O'Keeffe, and Omar M Yaghi. The chemistry and applications of metal-organic frameworks. Science, 341(6149):1230444, 2013.

[14] Christian S Diercks and Omar M Yaghi. The atom, the molecule, and the covalent organic framework. Science, 355(6328), 2017.

[15] Marc A Little and Andrew I Cooper. The chemistry of porous organic molecular materials. Advanced Functional Materials, page 1909842, 2020.

[16] Eric J Gosselin, Casey A Rowland, and Eric D Bloch. Permanently microporous metal-organic polyhedra. Chemical Reviews, 2020.

[17] Fei-Yan Yi, Dongxiao Chen, Meng-Ke Wu, Lei Han, and Hai-Long Jiang. Chemical sensors based on metal-organic frameworks. ChemPlusChem, 81(8):675-690, 2016.

[18] Lauren E Kreno, Kirsty Leong, Omar K Farha, Mark Allendorf, Richard P Van Duyne, and Joseph T Hupp. Metal-organic framework materials as chemical sensors. Chemical Reviews, 112(2):11051125, 2011.

[19] Pawan Kumar, Akash Deep, and Ki-Hyun Kim. Metal organic frameworks for sensing applications. TrAC Trends in Analytical Chemistry, 73:39-53, 2015.

[20] Michelle Woellner, Steffen Hausdorf, Nicole Klein, Philipp Mueller, Martin W Smith, and Stefan Kaskel. Adsorption and detection of hazardous trace gases by metal-organic frameworks. Advanced Materials, 30(37):1704679, 2018. 
[21] Hao Wang, William P Lustig, and Jing Li. Sensing and capture of toxic and hazardous gases and vapors by metal-organic frameworks. Chemical Society Reviews, 47(13):4729-4756, 2018.

[22] Xigui Liu, Danlian Huang, Cui Lai, Guangming Zeng, Lei Qin, Han Wang, Huan Yi, Bisheng Li, Shiyu Liu, Mingming Zhang, et al. Recent advances in covalent organic frameworks (cofs) as a smart sensing material. Chemical Society Reviews, 48(20):5266-5302, 2019.

[23] Malte Brutschy, Markus W Schneider, Michael Mastalerz, and Siegfried R Waldvogel. Porous organic cage compounds as highly potent affinity materials for sensing by quartz crystal microbalances. Advanced Materials, 24(45):6049-6052, 2012.

[24] MR Tchalala, PM Bhatt, KN Chappanda, SR Tavares, K Adil, Y Belmabkhout, A Shkurenko, A Cadiau, N Heymans, G De Weireld, G Maurin, KN Salama, and M Eddaoudi. Fluorinated mof platform for selective removal and sensing of $\mathrm{SO}_{2}$ from flue gas and air. Nature Communications, 10(1):1328, 2019.

[25] Rui-Biao Lin, Si-Yang Liu, Jia-Wen Ye, Xu-Yu Li, and Jie-Peng Zhang. Photoluminescent metalorganic frameworks for gas sensing. Advanced Science, 3(7):1500434, 2016.

[26] Mark D Allendorf, Ronald JT Houk, Leanne Andruszkiewicz, A Alec Talin, Joel Pikarsky, Arnab Choudhury, Kenneth A Gall, and Peter J Hesketh. Stress-induced chemical detection using flexible metal- organic frameworks. Journal of the American Chemical Society, 130(44):14404-14405, 2008.

[27] Hamish Yeung, G Yoshikawac, and K Shibab. Strain-based chemical sensing using metal-organic framework nanoparticles. Journal of Materials Chemistry A, 2020.

[28] Michael G Campbell, Sophie F Liu, Timothy M Swager, and Mircea Dinca. Chemiresistive sensor arrays from conductive $2 \mathrm{~d}$ metal-organic frameworks. Journal of the American Chemical Society, 137(43):13780-13783, 2015.

[29] Andrzej Gladysiak, Tu N Nguyen, Jorge AR Navarro, Matthew J Rosseinsky, and Kyriakos C Stylianou. A recyclable metal-organic framework as a dual detector and adsorbent for ammonia. Chemistry-A European Journal, 23(55):13602-13606, 2017.

[30] O Shekhah, J Liu, RA Fischer, and Ch Wöll. Mof thin films: existing and future applications. Chemical Society Reviews, 40(2):1081-1106, 2011.

[31] Yujing Zhang and Chih-Hung Chang. Metal-organic framework thin films: Fabrication, modification, and patterning. Processes, 8(3):377, 2020.

[32] Ivo Stassen, Nicholas Burtch, Alec Talin, Paolo Falcaro, Mark Allendorf, and Rob Ameloot. An updated roadmap for the integration of metal-organic frameworks with electronic devices and chemical sensors. Chemical Society Reviews, 46(11):3185-3241, 2017.

[33] C.K. O'Sullivan and G.G. Guilbault. Commercial quartz crystal microbalances - theory and applications. Biosensors and Bioelectronics, 14(8):663-670, 1999.

[34] Jay W Grate. Acoustic wave microsensor arrays for vapor sensing. Chemical Reviews, 100(7):26272648, 2000. 
[35] Rob Ameloot, Linda Stappers, Jan Fransaer, Luc Alaerts, Bert F Sels, and Dirk E De Vos. Patterned growth of metal-organic framework coatings by electrochemical synthesis. Chemistry of Materials, 21(13):2580-2582, 2009.

[36] Enrica Biemmi, Alexander Darga, Norbert Stock, and Thomas Bein. Direct growth of $\mathrm{Cu}_{3}(\mathrm{btc})_{2}\left(\mathrm{~h}_{2} \mathrm{O}\right)_{3} \cdot x \mathrm{~h}_{2} \mathrm{O}$ thin films on modified $\mathrm{qcm}$-gold electrodes-water sorption isotherms. Microporous and Mesoporous Materials, 114(1-3):380-386, 2008.

[37] Hiroki Yamagiwa, Seiko Sato, Tadashi Fukawa, Tsuyoshi Ikehara, Ryutaro Maeda, Takashi Mihara, and Mutsumi Kimura. Detection of volatile organic compounds by weight-detectable sensors coated with metal-organic frameworks. Scientific Reports, 4:6247, 2014.

[38] Min Tu, Suttipong Wannapaiboon, Kira Khaletskaya, and Roland A Fischer. Engineering zeoliticimidazolate framework (zif) thin film devices for selective detection of volatile organic compounds. Advanced Functional Materials, 25(28):4470-4479, 2015.

[39] Xueting Fang, Luyu Wang, Xiang He, Jiaqiang Xu, and Zhiming Duan. A 3d calcium spirobifluorene metal-organic framework: Single-crystal-to-single-crystal transformation and toluene detection by a quartz crystal microbalance sensor. Inorganic Chemistry, 57(4):1689-1692, 2018.

[40] Lakshmoji Kosuru, Adam Bouchaala, Nizar Jaber, and Mohammad I Younis. Humidity detection using metal organic framework coated on qcm. Journal of Sensors, 2016, 2016.

[41] Mohamed Rachid Tchalala, Youssef Belmabkhout, Karim Adil, Karumbaiah Nanaiah Chappanda, Amandine Cadiau, Prashant M Bhatt, Khaled Nabil Salama, and Mohamed Eddaoudi. Concurrent sensing of $\mathrm{CO}_{2}$ and $\mathrm{h}_{2} \mathrm{O}$ from air using ultramicroporous fluorinated metal-organic frameworks: Effect of transduction mechanism on the sensing performance. ACS Applied Materials \& Interfaces, 11(1):1706-1712, 2018.

[42] Xiaoliang Si, Chengli Jiao, Fen Li, Jian Zhang, Shuang Wang, Shuang Liu, Zhibao Li, Lixian Sun, Fen $\mathrm{Xu}$, and Zelimir Gabelica. High and selective $\mathrm{CO}_{2}$ uptake, $\mathrm{h}_{2}$ storage and methanol sensing on the amine-decorated 12-connected mof cau-1. Energy \& Environmental Science, 4(11):4522-4527, 2011.

[43] Benjamin Paschke, Achim Wixforth, Dmytro Denysenko, and Dirk Volkmer. Fast surface acoustic wave-based sensors to investigate the kinetics of gas uptake in ultra-microporous frameworks. ACS Sensors, 2(6):740-747, 2017.

[44] Alex L Robinson, Vitalie Stavila, Todd R Zeitler, Michael I White, Steven M Thornberg, Jeffery A Greathouse, and Mark D Allendorf. Ultrasensitive humidity detection using metal-organic framework-coated microsensors. Analytical Chemistry, 84(16):7043-7051, 2012.

[45] Vitalie Stavila, Christian Schneider, Curtis Mowry, Todd R Zeitler, Jeffery A Greathouse, Alex L Robinson, Julie M Denning, Joanne Volponi, Kirsty Leong, William Quan, et al. Thin film growth of nbo mofs and their integration with electroacoustic devices. Advanced Functional Materials, 26(11):1699-1707, 2016.

[46] Mohamed Eddaoudi, David B Moler, Hailian Li, Banglin Chen, Theresa M Reineke, Michael O'keeffe, and Omar M Yaghi. Modular chemistry: secondary building units as a basis for the 
design of highly porous and robust metal- organic carboxylate frameworks. Accounts of Chemical Research, 34(4):319-330, 2001.

[47] Zhenqiang Wang and Seth M Cohen. Postsynthetic modification of metal-organic frameworks. Chemical Society Reviews, 38(5):1315-1329, 2009.

[48] Peyman Z Moghadam, Aurelia Li, Seth B Wiggin, Andi Tao, Andrew GP Maloney, Peter A Wood, Suzanna C Ward, and David Fairen-Jimenez. Development of a cambridge structural database subset: a collection of metal-organic frameworks for past, present, and future. Chemistry of Materials, 29(7):2618-2625, 2017.

[49] Keith J Albert, Nathan S Lewis, Caroline L Schauer, Gregory A Sotzing, Shannon E Stitzel, Thomas P Vaid, and David R Walt. Cross-reactive chemical sensor arrays. Chemical reviews, 100(7):2595-2626, 2000.

[50] Arni Sturluson, Rachel Sousa, Yujing Zhang, Melanie T Huynh, Caleb Laird, Arthur HP York, Carson Silsby, Chih-Hung Chang, and Cory M Simon. Curating metal-organic frameworks to compose robust gas sensor arrays in dilute conditions. ACS Applied Materials \& Interfaces, 2020.

[51] Bettina Malnic, Junzo Hirono, Takaaki Sato, and Linda B Buck. Combinatorial receptor codes for odors. Cell, 96(5):713-723, 1999.

[52] Amine Bermak, Sofian Brahim Belhouari, Minghua Shi, Dominique Martinez, et al. Pattern recognition techniques for odor discrimination in gas sensor array. Encyclopedia of Sensors, 10:1-17, 2006.

[53] Julian W Gardner and Philip N Bartlett. A brief history of electronic noses. Sensors and Actuators B: Chemical, 18(1-3):210-211, 1994.

[54] Jenna Ann Gustafson and Christopher E Wilmer. Intelligent selection of metal-organic framework arrays for methane sensing via genetic algorithms. ACS Sensors, 4(6):1586-1593, 2019.

[55] Jenna A Gustafson and Christopher E Wilmer. Computational design of metal-organic framework arrays for gas sensing: Influence of array size and composition on sensor performance. The Journal of Physical Chemistry C, 121(11):6033-6038, 2017.

[56] Jenna A Gustafson and Christopher E Wilmer. Optimizing information content in mof sensor arrays for analyzing methane-air mixtures. Sensors and Actuators B: Chemical, 267:483-493, 2018.

[57] Brian A Day and Christopher E Wilmer. Genetic algorithm design of mof-based gas sensor arrays for c02-in-air sensing. Sensors, 20(3):924, 2020.

[58] Arni Sturluson, Melanie T Huynh, Alec R Kaija, Caleb Laird, Sunghyun Yoon, Feier Hou, Zhenxing Feng, Christopher E Wilmer, Yamil J Colón, Yongchul G Chung, et al. The role of molecular modelling and simulation in the discovery and deployment of metal-organic frameworks for gas storage and separation. Molecular Simulation, 45(14-15):1082-1121, 2019.

[59] Pierre C Sabatier. Past and future of inverse problems. Journal of Mathematical Physics, 41(6):4082-4124, 2000. 
[6o] Francisco Duarte Moura Neto and Antônio José da Silva Neto. An introduction to inverse problems with applications. Springer Science \& Business Media, 2012.

[61] Tanja Tarvainen Marko Vauhkonen and Timo Lähivaara. Inverse problems. In Mathematical modelling, pages 207-228. Springer, 2016.

[62] Charles W Groetsch and CW Groetsch. Inverse problems in the mathematical sciences, volume 52. Springer, 1993.

[63] Jarad A Mason, Thomas M McDonald, Tae-Hyun Bae, Jonathan E Bachman, Kenji Sumida, Justin J Dutton, Steven S Kaye, and Jeffrey R Long. Application of a high-throughput analyzer in evaluating solid adsorbents for post-combustion carbon capture via multicomponent adsorption of $\mathrm{CO}_{2}, \mathrm{n}_{2}$, and $\mathrm{h}_{2} \mathrm{O}$. Journal of the American Chemical Society, 137(14):4787-4803, 2015.

[64] Jarad A Mason, Mike Veenstra, and Jeffrey R Long. Evaluating metal-organic frameworks for natural gas storage. Chemical Science, 5(1):32-51, 2014.

[65] Hans Swenson and Nicholas P Stadie. Langmuir's theory of adsorption: A centennial review. Langmuir, 35(16):5409-5426, 2019.

[66] Yangzesheng Sun, Robert F Dejaco, and J Ilja Siepmann. Deep neural network learning of complex binary sorption equilibria from molecular simulation data. Chemical Science, 10(16):43774388, 2019.

[67] Sergei Igorevich Kabanikhin. Definitions and examples of inverse and ill-posed problems. Journal of Inverse and III-Posed Problems, 16(4):317-357, 2008.

[68] Mark Kac. Can one hear the shape of a drum? The American Mathematical Monthly, 73(4P2):1-23, 1966.

[69] MH Protter. Can one hear the shape of a drum? revisited. SIAM Review, 29(2):185-197, 1987.

[70] Carolyn Gordon and David Webb. You can't hear the shape of a drum. American Scientist, 84(1):46-55, 1996.

[71] Rodrigo Platte. Condition numbers and inverse problems. https://math.la.asu.edu/ rasurtg/talks/RTGintro_inv_problems.pdf. 2017.

[72] Andreas Schneemann, Volodymyr Bon, Inke Schwedler, Irena Senkovska, Stefan Kaskel, and Roland A Fischer. Flexible metal-organic frameworks. Chemical Society Reviews, 43(16):60626096, 2014.

[73] Krista S Walton and David S Sholl. Predicting multicomponent adsorption: 50 years of the ideal adsorbed solution theory. AIChE Journal, 61(9):2757-2762, 2015.

[74] Cory M Simon, Berend Smit, and Maciej Haranczyk. pyiast: Ideal adsorbed solution theory (iast) python package. Computer Physics Communications, 200:364-380, 2016.

[75] Alan L Myers and John M Prausnitz. Thermodynamics of mixed-gas adsorption. AlChE Journal, 11(1):121-127, 1965. 
[76] James F Epperson. An introduction to numerical methods and analysis. John Wiley \& Sons, 2013.

[77] Lloyd N Trefethen and David Bau III. Numerical linear algebra, volume 50. Siam, 1997.

[78] Gilbert Strang. Introduction to linear algebra, volume 5. Wellesley-Cambridge Press, 2016.

[79] Stephen S-Y Chui, Samuel M-F Lo, Jonathan PH Charmant, A Guy Orpen, and Ian D Williams. A chemically functionalizable nanoporous material [cu3 (tma) 2 (h20) 3] n. Science, 283(5405):11481150, 1999.

[80] Mohamed Eddaoudi, Jaheon Kim, Nathaniel Rosi, David Vodak, Joseph Wachter, Michael O'Keeffe, and Omar M Yaghi. Systematic design of pore size and functionality in isoreticular mofs and their application in methane storage. Science, 295(5554):469-472, 2002.

[81] Andrew R Millward and Omar M Yaghi. Metal- organic frameworks with exceptionally high capacity for storage of carbon dioxide at room temperature. Journal of the American Chemical Society, 127(51):17998-17999, 2005.

[82] Tim C Pearce. Odor to sensor space transformations in biological and artificial noses. Neurocomputing, 32:941-952, 2000.

[83] Pauli Virtanen, Ralf Gommers, Travis E. Oliphant, Matt Haberland, Tyler Reddy, David Cournapeau, Evgeni Burovski, Pearu Peterson, Warren Weckesser, Jonathan Bright, Stéfan J. van der Walt, Matthew Brett, Joshua Wilson, K. Jarrod Millman, Nikolay Mayorov, Andrew R. J. Nelson, Eric Jones, Robert Kern, Eric Larson, CJ Carey, IIhan Polat, Yu Feng, Eric W. Moore, Jake Vand erPlas, Denis Laxalde, Josef Perktold, Robert Cimrman, Ian Henriksen, E. A. Quintero, Charles R Harris, Anne M. Archibald, Antônio H. Ribeiro, Fabian Pedregosa, Paul van Mulbregt, and SciPy 1. 0 Contributors. SciPy 1.0: Fundamental Algorithms for Scientific Computing in Python. Nature Methods, 17:261-272, 2020. 